\title{
Pump and signal power combiners for high-power fiber amplifier applications
}

\author{
D. Sliwinska, ${ }^{*}$ P. Kaczmarek, and K. M. Abramski \\ Laser and Fiber Electronics Group, Wroclaw University of Technology, Wybrzeze Wyspianskiego 27, 50-370 \\ Wroclaw, Poland
}

Received February 05, 2015; accepted March 16, 2015; published March 31, 2015

\begin{abstract}
In this work we present the results of our research on a pump power combiner fabrication process in configuration $(\mathrm{N}+1) \times 1$. Our fabrication method is based on tapering a fiber bundle consisting of 6 multimode fibers and one signal fiber. We present the results on fabrication power combiners based on single mode fibers and large mode area fibers for the signal.
\end{abstract}

Pump power combiners are one of the most important components in all-fiber laser and amplifiers construction technique [1]. In 1988 Eli Snitzer et al. presented the first cladding-pumped fiber laser - based on double-clad fiber [2]. It appeared that double clad (DC) fibers are a very beneficial waveguide for high-power laser and amplifier constructions [3]. Since that time, several pumping techniques have been proposed. Three well known approaches are based on bulk optics and mechanical processing of fibers. They are a side-pumping method [45], through the v-grove [6], and an end-pumping method, which is the most popular one with efficiency reaching 70-85\% [7-8]. All these methods are very efficient, however, they are also very problematic. They all demand precise optical and mechanical adjustment, which makes the laser or amplifier setup construction very complicated and sensitive for external factors like the possibility of contamination and vibrations [9-10].

To avoid all this issues, a pump power combiner can be used. It is an all-fiber passive component which allows to effectively couple pump power and signal to an active double clad fiber. It consists of several fibers for the pump power at the input and one output passive double clad fiber. In the case of amplifier application, one of the input fibers is intended for signal transmission. This construction technique allows to make the laser or amplifier setup simple, robust, immune to external factors (e.g. contamination and vibration) and provide a very efficient coupling of pump power into an active doubleclad fiber [11-13]. A very important advantage in all-fiber construction is that the laser or amplified beam is not leaving the waveguide - core of the fiber. This allows to maintain high quality of the beam.

\footnotetext{
*E-mail: dorota.sliwinska@pwr.edu.pl
}

There were presented several approaches of power combiner fabrication techniques. One was presented by D. J. DiGiovanni et al. in 1999 [14]. The presented combiner was fabricated by bundling together multimode pump fibers with a signal fiber and tapering them down. The final diameter of the taper in the waist should be matched with the double clad fiber diameter. In this fabrication process a high tapering ratio (TR) can occur resulting in significant losses, because of mismatching mode field diameters. Therefore new fabrication techniques of pump power combiners are still being developed by scientific groups [10, 11, 15-19].

In this paper we present the results of our studies on pump power combiners fabrication in configuration $(\mathrm{N}+1) \times 1$. For a fabrication process, a 3 SAE LDS System (Large Diameter Splicer) was used [20]. The most important advantage of this splicer are three electrodes instead of conventional two. They make a so-called Ring Of Fire - ROF, so the tapered fiber or fiber bundle is evenly heated from each side. Additionally, the position of electrodes can be changed so the operation is possible on fibers with a diameter from $80 \mu \mathrm{m}$ to even $2 \mathrm{~mm}$. The splicer provides two methods of tapering - Fig. 1 .

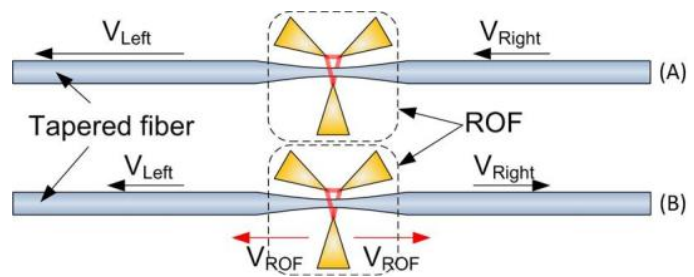

Fig. 1. Single direction (A) and bi-directional (B) tapering method.

Single directional tapering (Fig. 1A) is designed for capillary tubes, where the final shape of the taper has no influence on transmission. In this process the fiber is moving in one direction and the ROF remains in a central position. By increasing the velocity of one side of the fiber during heating by $\mathrm{ROF}$, it is possible to fabricate a taper with a programmed length of the waist, down-slope, up-slope, and waist diameter. A bi-directional tapering process (Fig. 1B) is designed for tapering of fibers and fiber bundles, where the shape of the fabricated structure has crucial influence on transmission efficiency. 
Our power combiners in configuration $(6+1) \times 1$ were fabricated using a bi-directional method of tapering. This method is more precise and allows to achieve very long fiber tapers with an extremely small waist diameter. In this method the fiber (or fiber bundle) is stretched while the $\mathrm{ROF}$ is heating it and scanning many times for proper distance. Controlling all tapering process parameters, like e.g. velocity and pull length of stretching, scanning velocity power and the distances of the ROF, allows to fabricate tapered fibers and fiber bundles with high transmission efficiency.

The first type of fabricated power combiner was based on single mode (SM) fibers dedicated for the $1550 \mathrm{~nm}$ signal. In configuration $(6+1) \times 1$ at the input were planned 6 multimode fibers $(105 / 125 \mu \mathrm{m})$ for the pump power and one SM fiber (SMF-28e) for the signal. The used capillary tube (TSP 530700, Optronis $\mathrm{GmbH}$ ) has an inner/external diameter of $530 / 650 \mu \mathrm{m}$, therefore it was firstly tapered down to $400 / 500 \mu \mathrm{m}$ with an almost $30 \mathrm{~mm}$ long waist. Then all seven input fibers were placed in a capillary tube, and the whole bundle was tapered down to the diameter of the output fiber. As an output fiber, a passive DC fiber 9/125 $\mu \mathrm{m}$ (GDF-1550 Nufern) was used. Figure 2 shows the cross sectional area of the described fiber bundle placed in a capillary tube. The total diameter of the bundle consisting of seven fibers is $375 \mu \mathrm{m}$ and it should be tapered down to the diameter of the output fiber, which is $125 \mu \mathrm{m}$. This gives TR equal to 3 , which means that the core of the signal fiber will be reduced from $8.2 \mu \mathrm{m}$ to less than $3 \mu \mathrm{m}$. This can cause critical losses in signal transmission.

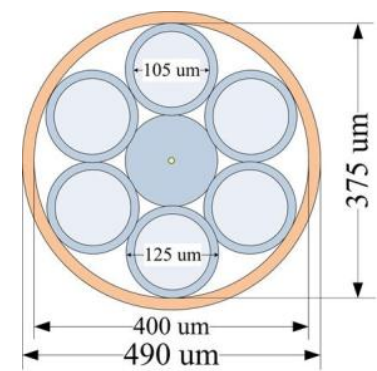

Fig. 2. Cross sectional area of simulated fiber bundle.

The computer simulation (using BeamPROP software) shows that tapering conventional SM fiber (in the fiber bundle) to a required final diameter will result in maximum signal transmission on the level of $65 \%$. Additionally, if taking into account possible imperfections in taper shape and some misalignments in splicing, the final transmission could be even lower.

The power combiner fabricated in the configuration described above, confirmed the simulation results and predictions. Signal transmission does not exceed $40 \%$. Additional losses are caused, as we assume, by a nonideal shape of the taper in comparison to simulation. The cleaving and splicing process of a bundle to a DC fiber can further increase losses.

To reduce signal losses, the taper ratio needed to be decreased. We decided to use a $9 / 80 \mu \mathrm{m}$ (Nufern) SM fiber instead of conventional 8.2/125. Using the signal fiber with a reduced clad diameter, but with the same mode field diameter, could effectively improve signal transmission. Simulations have shown that tapering a $9 / 80 \mu \mathrm{m}$ single mode fiber to a needed diameter, can provide signal transmission on the level above $95 \%$. However, one inconvenience appeared - placing six $105 / 125 \mu \mathrm{m}$ fibers and one $9 / 80 \mu \mathrm{m}$ will cause the wrong placement of all fibers in a capillary tube.

Therefore the first step of the fabrication process was to taper down seven multimode fibers to $80 \mu \mathrm{m}$ in the waist. The length of the down-slope of taper was about $17 \mathrm{~mm}$ and the waist length $\sim 34 \mathrm{~mm}$. The used capillary tube was tapered down to $250 / 300 \mu \mathrm{m}$ with an almost $30 \mathrm{~mm}$ long waist. The capillary was then broken at one end of the waist and seven multimode fibers were placed inside it. The central multimode fiber was then switched to a $9 / 80 \mu \mathrm{m}$ SM fiber. The earlier cut-off part of the capillary was then attached to the part filled with fibers. The final tapering process involving the total capillary waist length, and final diameter, including the thickness of the capillary tube, was $145 \mu \mathrm{m}$.

Figure 3 shows the recorded profile of the tapered fiber bundle and the cross section area with a marked diameter of the fiber bundle inside the capillary tube (about $105 \mu \mathrm{m})$. The TR of the fiber bundle was decreased from 3 to 2.4 .

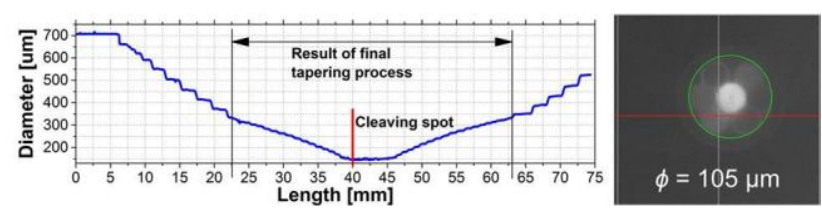

Fig. 3. Recorder profile of tapered fiber bundle based on SM fibers for signal transmission and cross section area recorded after cleaving.

The tapered bundle was then cleaved in the LDS System in the waist and spliced to a passive double clad fiber.

During the splicing process, transmission of signal was controlled in order to achieve the best coupling of signal between the input and output fiber. The transmission of $1550 \mathrm{~nm}$ signal after splicing to a double clad fiber achieved the level of about $70 \%$ (attenuation $1.7 \mathrm{~dB}$ ). It is lower than simulated transmission level, because of some imperfections of tapering process, cleaving and splicing comparing to simulated ideal linearly tapered structure and cleaved without angular misalignment. The coupling efficiency of pump power was measured for each single multimode port and varies from 72.2 to $75.5 \%$.

The fabricated structure was then placed in an aluminum housing, in order to provide proper heat dissipation, and it 
was tested in the case of long term operation. After 30 minutes of transmission (nearly $30 \mathrm{~W}$ of pump power with a coupling efficiency of $74 \%$ ), the temperature of the combiner remained at $60^{\circ} \mathrm{C}$ and did not exceed in the next hour. The backward propagation of the signal was also measured, which can occur in a laser or amplifier configuration. This phenomenon can cause damage of the setup or used pump source. In this measurement at the output of the combiner, a $1550 \mathrm{~nm}$ signal with a power of $16 \mathrm{~mW}$ was launched. The measured transmission at the input pump ports was successfully suppressed by $25 \mathrm{~dB}$.

The second fabricated type of $(6+1) \times 1$ power combiner configuration was based on large mode area fibers (LMA). As pump ports were used $105 / 125 \mu \mathrm{m}$ multimode fibers as well. As a signal input fiber was used a 20/125 $\mu \mathrm{m}$ LMA fiber (LMA-GDF-20/130-M, Nufern), and as an output port - a 25/300 $\mu \mathrm{m}$ double clad fiber (LMA-GDF-25/300, Nufern). In the case of this type of combiner no tapering of multimode fibers is necessary. Also TR of tapering an empty capillary tube is much lower $(\mathrm{TR}=1.3)$, because it was tapered down to $400 / 500 \mu \mathrm{m}$. The procedure of inserting fibers into the capillary tube and switching the central fiber to a signal fiber is the same as previously. The only difference is the final diameter of the tapered fiber bundle - about $300 \mu \mathrm{m}$. The TR of the final tapering process in this case is much lower - 1.7. Recorded profile of the tapered fiber bundle and cross section of fibers inside the capillary, with a diameter of $255 \mu \mathrm{m}$ is shown in Fig. 4.

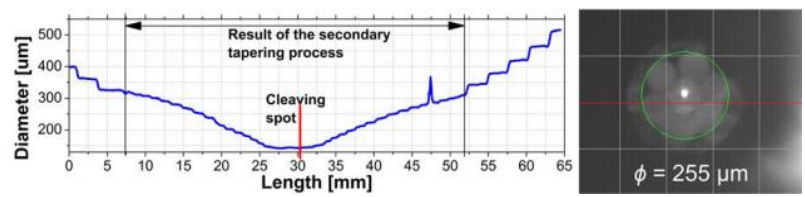

Fig. 4. Recorded profile of tapered fiber bundle based on LMA fibers for signal transmission and recorded cross section area after cleaving.

The transmission of a $1550 \mathrm{~nm}$ signal during the splicing was monitored to achieve the best coupling of the input and output fiber core of the combiner. After splicing the signal transmission at a level of nearly $80 \%$ was recorded. The measured coupling efficiency of pump power for each single multimode port varied from 97 to $99 \%$.

As in the previous combiner (based on SM signal fibers), also this one was placed in an aluminum housing. It was also tested in case of long term operation. During an hour transmission of $30 \mathrm{~W}$ pump power with a $98 \%$ coupling efficiency, the temperature of the combiner did not exceed $50^{\circ} \mathrm{C}$. The measurements of backward propagation have shown that the $1550 \mathrm{~nm}$ signal is successfully suppressed in all six multimode ports by $17 \mathrm{~dB}$.

In conclusion, we have shown our power combiner fabrication method using a three-electrode LDS system.
Our power combiners were performed in configuration $(6+1) \times 1$ using two types of signal fibers: single mode and large mode area fibers. In the case of a power combiner based on SM fibers, the signal transmission at $70 \%$ level and pump transmission at $72.2-75.5 \%$ were achieved. Further improvements are possible by optimizing the fabrication process. For a power combiner based on LMA fibers we have achieved signal transmission at $80 \%$ level and pump transmission at $97-99 \%$ level, which is a very satisfactory result. Also in the case of this type of combiner, some improvements are possible, especially for signal transmission. LMA fibers are much more sensitive to angular misalignments than SM fibers, because of the larger mode field diameter. Thus improving the cleaving process, could allow us to increase signal transmission.

The work presented in this paper was supported by Wroclaw Research Center EIT+ under the project "The Application of Nanotechnology in Advanced Materials", NanoMat (POIG.01.01.02-02-002/08) financed by the European Regional Development Fund (Innovative Economy Operational Programme, 1.1.2)

\section{References}

[1] D. Noordegraaf et al., Proc. SPIE 7914, 79142 (2011).

[2] E. Snitzer, H. Po, F. Hakimi, R. Tumminelli, B.C. McCollum, Opt. Fiber Sensor Topical Meeting, New Orleans, LA (1988).

[3] M.N. Zervas, C.A. Codemard, IEEE J. Selected Topics in Quantum Electronics 20(5), 219 (2014)

[4] F. Hakimi, H. Hakimi, Technical Digest: Lasers and Electro-Optics Conference (CLEO 2001), 116 (2001).

[5] J.P. Koplow, S.W. Moore, D.A.V. Kliner, IEEE J. Selected Topics in Quantum Electr. 39, 4 (2003).

[6] Ch. Li, D. Shen, J. Song, N. S. Kim, K. Ueda, Technical Digest: Lasers and Electro-Optics Conference (CLEO 1999), 3 (1999).

[7] J. Swiderski, A. Zając, M. Skorczakowski, Opto-Electr. Rev. 15, 2, (2007).

[8] Y. Jeong et al., IEEE J. Selected Topics in Quantum Electronics 13, 3 (2007).

[9] W. Shi, Q. Fang, X. Zhu, R.A. Norwood, N. Peyghambarian, Appl. Opt. 53, 28 (2014).

[10] D. J.Richardson, J. Nilsson, W.A. Clarkson, J. Opt. Soc. Am. B 27, 11 (2010).

[11] F. Gonthier et al., Fiber Lasers: Technology, Systems and Applications, 266-276 (2004).

[12] A. Braglia, A. Califano, Y. Liu, G. Perrone, Intern. J. Modern Phys. B 28, 12 (2014).

[13] G. Sobon, P. Kaczmarek, D. Sliwinska, J. Sotor, K. Abramski, IEEE J. Selected Topics in Quantum Electronics 20, 5 (2014).

[14] D. J. Di Giovanni, A.J. Stentz, U.S. Patent 5,864,644 A (1999).

[15] A. Braglia, A. Califano, Y. Liu, G. Perrone, Intern. J. Modern Phys. B 28, 12 (2014).

[16] J. K. Kim et al., Opt. Expr. 18, 12 (2010).

[17] B. Sévigny, P. Poirier, M. Faucher, Proc. SPIE 7195, 719523 (2009).

[18] A. Kosterin, V. Temyanko, M. Fallahi, M. Mansuripur, Technical Digest: OFC/NFOEC, 2 (2005).

[19] P. Koška, Y. Baravets,P. Peterka, J. Bohata, M. Písařík, Appl. Opt. 54, 4 (2015).

[20] R. Wiley, B. Clark, Proc. SPIE 7914, 79140F (2011). 